\title{
Endoscopic Biliary Stenting for Portal Biliopathy Perforating Paracholedochal Collateral: A Rare Complication
}

\author{
Kartik Goyal ${ }^{1}$ Vaibhav Kumar Varshney2, $\quad$ Sabir Hussain ${ }^{1} \quad$ Pawan Kumar Garg $^{3} \quad$ Narender Bhargava $^{1}$ \\ ${ }^{1}$ Department of Gastroenterology, Mathura Das Mathur Hospital, \\ Jodhpur, Rajasthan, India \\ 2 Department of Surgical Gastroenterology, All India Institute of \\ Medical Sciences, Jodhpur, Rajasthan, India \\ Address for correspondence Dr. Vaibhav Kumar Varshney, MCH, \\ Department of Surgical Gastroenterology, All India Institute of \\ Medical Sciences, Basni Industrial Area, Phase II, Jodhpur, \\ Rajasthan 342005, India (e-mail: drvarshney09@gmail.com).
}

${ }^{3}$ Department of Diagnostic and Interventional Radiology, All India Institute of Medical Sciences, Jodhpur, Rajasthan, India

\begin{abstract}
Keywords

- EHPVO

- portal biliopathy

- paracholedochal plexus

- stent

Extrahepatic portal venous obstruction (EHPVO) usually presents with upper gastrointestinal bleed in the first decade of life. Symptomatic portal hypertensive biliopathy is seen in a minority of patients with EHPVO. With use of endoscopic intervention, biliary drainage is maintained in these patients. Various procedural complications have been linked while performing endoscopic retrograde cholangiography and stenting; however, these are managed conservatively. Here, we are highlighting a case of EHPVO with symptomatic portal biliopathy in which the patient bled from paracholedochal collateral after biliary stenting and was managed successfully with a multidisciplinary approach.
\end{abstract}

\section{Introduction}

Extrahepatic portal venous obstruction (EHPVO) is characterized by a chronic obstruction of extrahepatic portal vein, and usually presents in childhood with signs of portal hypertension. ${ }^{1}$ The diagnosis of EHPVO is primarily the clinical features of portal hypertension (PHT) without any evidence of liver dysfunction and is supported by the presence of portal cavernoma on abdominal ultrasonography (USG) with Doppler examination.

Portal biliopathy (PB) is seen in 80 to $100 \%$ of patients with EHPVO. However, 62 to $95 \%$ of these patients ${ }^{2}$ are asymptomatic for PB, and present with the features of PHT. Symptoms of PB include cholestatic jaundice, biliary colic, recurrent cholangitis, choledocholithiasis, and abnormal liver function tests showing obstructive pattern.

Endoscopic retrograde cholangiopancreatography (ERCP) with stenting is usually required in $\mathrm{PB}$ presenting with cholangitis. ${ }^{2}$ ERCP and stent-induced bleeding from the venous plexus in PB is extremely rare.,4 Here, we present a case of EHPVO with symptomatic PB who underwent biliary stenting which led to torrential bleeding from paracholedochal collateral, and discuss its management along with a brief review of the relevant literature.

\section{Case Report}

A 31-year-old male presented with right upper abdominal pain, high-grade fever, and progressive jaundice of 1 month duration. On examination, he was icteric, with moderate splenomegaly and tenderness in right hypochondrium. He was a known case of EHPVO diagnosed 15 years ago and was on secondary prophylaxis with $\beta$-blockers for variceal bleeding. He had undergone an uneventful open cholecystectomy 4 months ago for symptomatic cholelithiasis. With the background of EHPVO, PB was suspected and the patient was evaluated accordingly.

Investigation revealed leucocytosis $\left(19,700 / \mathrm{mm}^{3}\right)$, conjugated hyperbilirubinemia $(13.57 \mathrm{mg} / \mathrm{dL})$, and elevated alkaline phosphatase $(311 \mathrm{IU} / \mathrm{L})$. USG of the abdomen was suggestive of portal cavernoma and dilated common bile duct (CBD) with echogenic sludge within. Magnetic resonance 
imaging (MRI) abdomen with magnetic resonance cholangiopancreatography (MRCP) revealed a smooth narrowing at the lower end of CBD with upstream dilatation of CBD $(21 \mathrm{~mm})$ and intrahepatic biliary radicals (IHBR) with few soft calculi (2-4 mm). Upper gastrointestinal (UGI) endoscopy revealed eradicated esophageal varices and no gastric varices. In view of these findings corroborating the diagnosis of $\mathrm{PB}$, and in the presence of cholangitis, the patient underwent ERCP with a plan of biliary stenting. Selective CBD cannulation was done and cholangiogram revealed dilated IHBR with grossly dilated CBD, except narrowed distal segment. Minor bleed was reported after papillotomy done using pull type papillotome, which resolved spontaneously. Stones were extracted with the help of extraction balloon and a $10 \mathrm{Fr}$ $\times 10 \mathrm{~cm}$ straight plastic stent was placed. Immediately, a gush of blood was seen from ampulla into the duodenum, with the stent in situ. The patient developed sudden hypotension, and the procedure was terminated and the patient shifted to the intensive care unit (ICU).

The patient was resuscitated with intravenous fluids, inotropes, terlipressin, and 2 units of packed red blood cells transfusion. Nasogastric tube was placed, which showed frank blood mixed with bile. Parenteral broad-spectrum antibiotics and injectable tranexamic acid were instituted. The patient became hemodynamically stable in 8 to 10 hours with these measures.

A triphasic computed tomogram (CT) of the abdomen with thin-slice multiplanar reconstruction for vascular channels was performed. Noncontrast CT abdomen ( $\mathbf{- F i g . ~ 1 a ) ~ s h o w e d ~}$ contrast injected during ERCP still present within dilated IHBR. Biliary stent was away from the hepatic artery branches with no active contrast extravasation, blush, or pseudoaneurysm seen (-Fig. 1b). Portal venous phase (-Fig. 2a,b) showed the portal vein replaced with multiple paracholedochal collaterals with dilated CBD. Tip of the biliary stent was seen within one of the large paracholedochal collateral vessels.

Percutaneous transhepatic biliary drainage (PTBD) was done with external catheter placement at hilum to decompress biliary system and to avoid displacing the hematoma around collateral puncture site (-Fig. 3). The PTBD catheter did not show any further bleeding, and the nasogastric

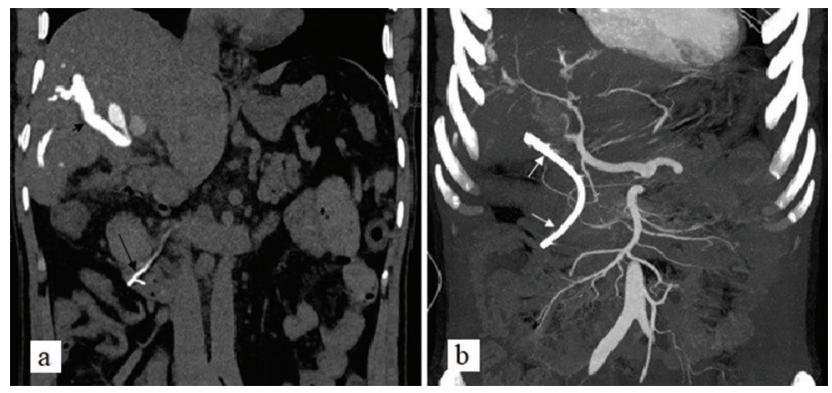

Fig. 1 (a) Noncontrast CT abdomen in coronal plane showing contrast-filled dilated intrahepatic biliary radicles (small black arrow). The lower end of common bile duct stent (large black arrow) is also seen. (b) Coronal maximum intensity projection in arterial phase showing biliary stent (white arrow) away from hepatic artery branches with no active extravasation or pseudoaneurysm seen.

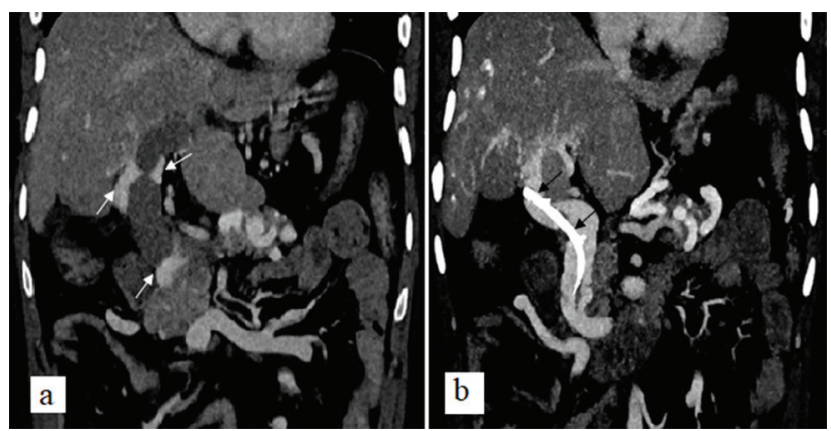

Fig. 2 CECT abdomen during portal venous phase in coronal plane showing multiple paracholedochal collaterals (white arrow) with dilated common bile duct. Tip of biliary stent (black arrow) is misplaced and seen within one of the larger paracholedochal collateral.

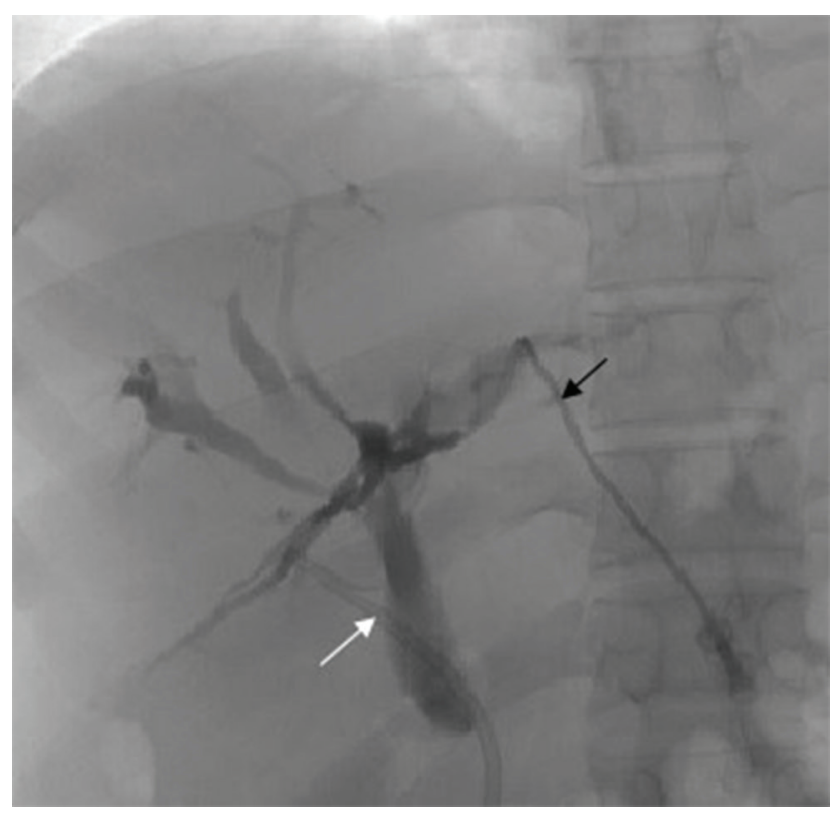

Fig. 3 Cholangiogram taken through an external biliary catheter (black arrow) placed from the left side biliary system into the right side showing mild dilatation of intrahepatic biliary radicals and common bile duct (CBD). Biliary stent seen within CBD in the lower part and misplaced out of CBD (white arrow) in the upper part.

drainage also showed clear bilious drainage after 24 hours. The patient's inotropes were tapered off and stopped, hemoglobin improved, and bilirubin showed a decreasing trend over the next 72 hours. After 7 days, ERCP was repeated and in situ stent was removed and $8 \mathrm{Fr} \times 10 \mathrm{~cm}$ double pigtail stent was placed into CBD. The procedure was uneventful. Two days later, the PTBD catheter was clamped, and with continued decreasing level of bilirubin, it was removed. The patient was subsequently discharged after cholangitis had resolved. On follow-up visit at 3 months, the patient was asymptomatic with no further episodes of GI bleed or cholangitis.

\section{Discussion}

Portal biliopathy (PB) is reported in 80 to $100 \%$ cases of EHPVO but presents with symptomatic biliary obstruction in only 5 to $14 \%{ }^{2}$ The mechanisms postulated to cause PB 
are dilated epicholedochal and paracholedochal venous plexuses, called portal cavernoma, causing extrinsic compression of the extrahepatic bile ducts; the formation of new vessels and connective tissue resulting in fibrosis around the ducts; and ischemic changes in bile duct. ${ }^{5}$ These processes may lead to cholestasis, biliary stricture, cholangitis, and choledocholithiasis.

Symptomatic PB is a definite indication for intervention endoscopic or surgical. Endoscopic interventions include sphincterotomy, stone extraction, biliary stenting, stricture dilatation, and mechanical lithotripsy. ${ }^{6}$ Surgical portosystemic shunt is done primarily to decompress the portal cavernoma and release pressure on CBD, and if symptoms persist then endobiliary interventions are utilized. However, surgical biliary drainage, either choledochoduodenostomy or hepaticojejunostomy, may be needed for persistent or recurrent stricture. ${ }^{7}$ In our case, due to the presence of splenic vein and superior mesenteric vein thrombosis with no shuntable varix, and in view of cholangitis, endoscopic management was chosen as the primary modality for management.

The overall success rate of endotherapy in portal biliopathy is 70 to $100 \%{ }^{2,8}$ Complications like bleeding, hemobilia, and pancreatitis have been reported with ERCP in the presence of PB. Most of these are managed conservatively, and further intervention-endoscopic, percutaneous, or surgical-is infrequently required. ${ }^{8}$ While performing ERCP in $\mathrm{PB}$, bleeding is encountered either during sphincterotomy, balloon dilatation, or while stent removal. ${ }^{3,4}$ Bleeding at the time of stent insertion due to migration of stent tip into a collateral vessel, as seen in our case, has not been reported in literature as per our knowledge.

ERCP-induced bleeding is usually self-limiting or can be easily controlled endoscopically in the same sitting without significant hemodynamic changes. Hemorrhagic shock, as seen in our case, is unusual, and should be managed as a case of UGI bleeding with fluid resuscitation, inotropes, splanchnic vasoconstrictors, and blood transfusion. In such scenario of cholestasis with coagulopathy, risk of massive bleeding due to bilioportal collateral fistula, and chances of bilhemia due to associated obstructive jaundice, the stent should not be removed initially. PTBD helps reduce the biliary pressure, decrease the level of bilirubin, and improves cholestasis and coagulopathy. Similar approach by endoscopic nasobiliary drainage (ENBD) has been utilized in the past for bridging period of acute hemorrhagic episode. ${ }^{9}$

As per our knowledge, this is the first reported instance of biliary stent-induced massive hemobilia due to injury to a pericholedochal collateral vessel. This case highlights various important aspects in managing such patients: (a) Utmost care while performing ERC stenting in symptomatic PB without portosystemic shunt, (b) importance of CT portal venography and EUS to delineate the biliary plexus collaterals in such patients, (c) biliary drainage with either PTBD or ENBD to improve cholestasis if hemobilia occurs, and (d) managing such patients in ICU with a multidisciplinary approach.

\section{Sources of Funding}

None.

\section{Conflict of Interest}

All authors declare that they have no conflict of interest.

\section{References}

1 Sarin SK, Agarwal SR. Extrahepatic portal vein obstruction. Semin Liver Dis 2002;22(1):43-58

2 Dhiman RK, Behera A, Chawla YK, Dilawari JB, Suri S. Portal hypertensive biliopathy. Gut 2007;56(7):1001-1008

3 Al-Akwaa AM, Elsadig M, Al-Fayaa AE, Al-Shehri MD. Portal hypertensive biliopathy presents with massive bleeding during ERCP after balloon sphincteroplasty in a noncirrhotic saudi sickler patient. Case Rep Med 2017;2017:4163919

4 Layec S, D'Halluin PN, Pagenault M, Bretagne JF. Massive hemobilia during extraction of a covered self-expandable metal stent in a patient with portal hypertensive biliopathy. Gastrointest Endosc 2009;70(3):555-556, discussion 556

5 Dhiman RK, Puri P, Chawla Y, et al. Biliary changes in extrahepatic portal venous obstruction: compression by collaterals or ischemic? Gastrointest Endosc 1999;50(5):646-652

6 Chandra R, Kapoor D, Tharakan A, Chaudhary A, Sarin SK. Portal biliopathy. J Gastroenterol Hepatol 2001;16(10):1086-1092

7 Agarwal AK, Sharma D, Singh S, Agarwal S, Girish SP. Portal biliopathy: a study of 39 surgically treated patients. HPB (Oxford) 2011;13(1):33-39

8 Oo YH, Olliff S, Haydon G, Thorburn D. Symptomatic portal biliopathy: a single centre experience from the UK. Eur J Gastroenterol Hepatol 2009;21(2):206-213

9 Mutignani M, Shah SK, Bruni A, Perri V, Costamagna G. Endoscopic treatment of extrahepatic bile duct strictures in patients with portal biliopathy carries a high risk of haemobilia: report of 3 cases. Dig Liver Dis 2002;34(8):587-591 